\title{
COMPARATIVE STUDY OF THE DIFFERENT VERSIONS OF THE GENERAL IMAGE QUALITY EQUATION
}

\author{
A. Q. Valenzuela ${ }^{1}$, J. C. G. Reyes ${ }^{2}$ \\ ${ }^{1}$ Chilean Air Force, Space Affairs Sub Directorate, Santiago de Chile - avalenzuela@fach.mil.cl \\ ${ }^{2}$ Chilean Air Force, Space Operations Squadron, Santiago de Chile - jcreyesg@fach.mil.cl
}

KEY WORDS: General image quality equation, image enhancement, image interpretability.

\begin{abstract}
:
The General Image Quality Equation (GIQE) is an analytical tool derived by regression modelling that is routinely employed to gauge the interpretability of raw and processed images, computing the most popular quantitative metric to evaluate image quality; the National Image Interpretability Rating Scale (NIIRS). There are three known versions of this equation; GIQE 3, GIQE 4 and GIQE 5, but the last one is scarcely known. The variety of versions, their subtleties, discontinuities and incongruences, generate confusion and problems among users. The first objective of this paper is to identify typical sources of confusion in the use of the GIQE, suggesting novel solutions to the main problems found in its application and presenting the derivation of a continuous form of GIQE 4, denominated GIQE 4C, that provides better correlation with GIQE 3 and GIQE 5. The second objective of this paper is to compare the predictions of GIQE 4C and GIQE 5, regarding the maximum image quality rating that can be achieved by image processing techniques. It is concluded that the transition from GIQE 4 to GIQE 5 is a major paradigm shift in image quality metrics, because it reduces the benefit of image processing techniques and enhances the importance of the raw image and its signal to noise ratio.
\end{abstract}

\section{INTRODUCTION}

The National Image Interpretability Rating Scale (NIIRS) was developed in the 1970's under the auspice of the U.S.A. Imagery Resolution Assessments and Reporting Standards (IRARS) Committee (Irvine, 1997).

NIIRS is a ten-level integer scale (from 0 to 9), that allows image interpreters to rank an image according to its interpretability defined as the usefulness to perform certain specific Detection, Recognition and Identification (DRI) tasks. The complexity of these tasks increases with the rating scale, for example regarding automobiles, an image with rating scale 6 (NIIRS level 6), allows their classification as either sedan or station wagons, whereas a NIIRS level 8 image, allows the detection of their windshield wipers.

The General Image Quality Equation (GIQE) is a mathematical tool that allows the NIIRS rating of an image to be computed as a function of parameters that describe sensor performance during image capture and, eventually, parameters that characterize processing techniques applied to the raw image.

All GIQE versions are obtained by regression analysis; a set of images acquired by sensors with known capture and processing parameters is selected and each image is assigned a NIIRS rating by image analysts. A "general" equation that models these ratings as a function of the capture and - eventually - processing parameters is then derived.

Whereas the original NIIRS had a discrete integer rating, the GIQE allows the computation of decimal NIIRS values with a standard error of approximately 0.3 NIIRS units. The quality perception threshold is about 0.1 NIIRS units; differences of this order are barely perceptible (Leachtenauer, 1997).

The first two versions of the General Image Quality Equation (GIQE 3 and GIQE 4) were developed in the 1980s for the intelligence community. These versions were not available for public use and cannot be discussed as their "documentation no longer exists" (Harrington, 2015).
GIQE 3 was developed in 1992 and released in 1994 for the Unmanned Aerial Vehicle (UAV) and sensor community (Griffith, 2012) through a user's guide that had restricted release whereas GIQE 4 was developed in 1996 for the commercial space imaging industry and released in 1997 through an open paper in the Applied Optics journal (Leachtenauer, 1997).

GIQE 3 and 4 were developed to rate hardcopy images whose processing parameters were already fixed and known, these equations rate the quality of either enhanced (processed) or "raw" (unprocessed; unenhanced) images, as a function of their processing parameters. These equations are also used to predict the maximum increase in NIIRS rating of the raw image due to image sharpening $(\triangle \mathrm{NIIRS})$, a common processing technique used to enhance image quality (Fiete, 2010).

GIQE 5 was released in 2015 by the National GeospatialIntelligence Agency (NGA) through a paper available in its web site (Harrington, 2015). This equation was developed to gauge softcopy images whose processing parameters are interactively selected by the image analyst; it differs from the previous two versions because it only computes a single NIIRS rating of a "well enhanced" image as a function of its unenhanced parameters without considering processing details. This version is scarcely known and it cannot be used to predict $\triangle$ NIIRS, for these reasons GIQE 4 is still widely used, although declared "obsolete" by the NGA (Harrington, 2015).

The NIIRS rating is a key image quality parameter of an electro optical sensor. The authors of this paper had to compare sensor designs from various satellite companies, and found that the specified GIQE NIIRS ratings were not always coherent with the expected sensor performance. An in depth investigation into this matter, lead to the conclusion that this lack of coherency was originated by the following four types of problems.

The first problem is that the GIQE has three known versions, and that each version gives different NIIRS ratings, creating confusion among users on which equation must be used. For example; a typical error is to use GIQE 3 to gauge satellite images when in this case GIQE 4 should be employed. For fixed GIQE parameters, the NIIRS rating of GIQE 3 is about 0.3 points higher 
than the one of GIQE 4; an attractive property for image marketing purposes. This difference is presumably due to the fact that interpretability is better when viewing and object from the side than from above.

The second problem is that some parameters used in the GIQE have peculiar definitions, so they are sometimes incorrectly computed by the users. For example; the GIQE "Signal to Noise Ratio" ("SNR") is actually a Signal Difference to Noise Ratio (SDNR) that can be much smaller than the usual SNR. In this paper, when referring to the GIQE parameter as presented in the literature, we use the "SNR" acronym within quotation marks.

The third problem is that the most popular version, GIQE 4, uses two different equations ( $4 \mathrm{a}$ and $4 \mathrm{~b}$ ) that predict different quality ratings at their point of transition. Thus, from a mathematical point of view, GIQE 4 is a discontinuous function leading to absurd results at or near the transition point.

The fourth problem is that the maximum NIIRS rating predicted by GIQE 4 is substantially larger than GIQE 5 prediction.

The first objective of this paper is to provide some guidelines and suggestions to solve the first three problems, with the purpose to help GIQE users to get meaningful results from the different versions of this equation. The third problem is solved by finding a continuous form of GIQE 4 (GIQE 4C).

The second objective of this paper is to compare GIQE 4C and GIQE 5 results, regarding the maximum image quality rating that can be achieved by image processing techniques.

\section{GENERAL IMAGE QUALITY EQUATION}

\subsection{GIQE 4 have Versions 3 and 4}

Versions 3 and the same form:

$$
\begin{aligned}
\text { NIIRS }_{\mathrm{i}}=\mathrm{A}+\mathrm{B} \cdot \log \left(\mathrm{GSD}_{\mathrm{i}}\right) & +\mathrm{C} \cdot \log (\mathrm{RER})+ \\
& +\mathrm{D} \cdot \mathrm{H}+\frac{\mathrm{E} \cdot \mathrm{G}}{\mathrm{SDNR}}
\end{aligned}
$$

where $\mathrm{A}, \mathrm{B}, \mathrm{C}, \mathrm{D}, \mathrm{E}=$ numerical constants defined below $\log (\mathrm{x})=$ base $10 \operatorname{logarithmic}$ function of $\mathrm{x}$ $\mathrm{i}=$ symbol 3 or 4 that identifies the GIQE version GSD $=$ Ground Sampling Distance $\mathrm{GSD}_{\mathrm{i}}=$ GSD used in GIQE 3 or 4 RER= Relative Edge Response used in GIQE 3 and 4 $\mathrm{H}=$ Height overshoot

$\mathrm{G}=$ Sharpening filter gain

SDNR $=$ Signal Difference to Noise ratio

The five constants are defined in Table 1 for GIQE 3, GIQE 4a (valid for RER < 0.9 ) and GIQE $4 \mathrm{~b}$ (valid for RER $\geq 0.9$ ), assuming that GSD is expressed in meters. Note that GIQE $4 \mathrm{a}$ and GIQE $4 \mathrm{~b}$ together define GIQE 4, and so they must not be considered different versions of the GIQE.

\begin{tabular}{|l|c|c|c|c|c|}
\hline Equation & \multicolumn{1}{c|}{ A } & \multicolumn{1}{c|}{ B } & \multicolumn{1}{c|}{ C } & \multicolumn{1}{c|}{ D } & \multicolumn{1}{c|}{ E } \\
\hline GIQE 3 & 6.514 & -3.32 & 3.32 & -1.48 & -1 \\
\hline GIQE 4a & 5.210 & -3.16 & 2.817 & -0.656 & -0.344 \\
\hline GIQE 4b & 4.955 & -3.32 & 1.559 & -0.656 & -0.344 \\
\hline
\end{tabular}

Table 1. GIQE 3 and 4 coefficients for GSD in metric units

The A coefficient is different if the GSD is expressed in inches, a possible source of confusion that leads to erroneous results.
The most typical source of error in the use of the GIQE, is to confuse the Signal to Noise Ratio (SNR) with the required Signal Difference to Noise Ratio (SDNR). We presume that this confusion is due to the fact that published versions of GIQE (Leachtenauer, 1997), (Harrington, 2015) use the acronym "SNR" to denote the SDNR. To avoid this confusion we use the SDNR acronym in the GIQE, provide formal definitions for SNR and SDNR (Equations 18 and 19), and derive a simple equation that relates these two parameters (Equation 20).

GIQE 4 coefficients were derived by regression analysis of a set of 359 images which covered the range of parameters indicated in Table 2 (Leachtenauer, 1997).

\begin{tabular}{|c|c|c|c|}
\hline Parameter & Minimum & Maximum & Mean \\
\hline GSD $(\mathrm{m})$ & 0.08 & 2.03 & 0.52 \\
\hline RER & 0.2 & 1.3 & 0.92 \\
\hline H & 0.9 & 1.9 & 1.3 \\
\hline G & 1 & 19 & 10.7 \\
\hline SDNR & 2 & 130 & 52 \\
\hline
\end{tabular}

Table 2. GIQE 4 range of parameters

In what follows the five parameters of the GIQE versions 3 and 4 are analysed in detail.

\subsubsection{Ground Sampling Distance}

The GSD for GIQE 3 is computed in a plane orthogonal to the Line Of Sight (LOS) as:

$$
\mathrm{GSD}_{\mathrm{OP}}=\frac{\mathrm{p}}{\mathrm{f}} \cdot \mathrm{R}
$$

where $\quad p=$ pixel pitch of detector

$\mathrm{f}=$ focal length of the sensor

$\mathrm{R}=$ slant range from sensor to the target

The previous definition of GSD in the Orthogonal Plane (OP) is applicable to airborne sensors which detect targets at elevation angles lower than $10^{\circ}$ (Harrington, 2015). In this case the resolution in the vertical plane is the one relevant for the DRI tasks, changes of a few degrees in the elevation angle will not make much difference, so this angle is not included in GIQE 3.

The GSD for GIQE 4 is computed as the ground plane projection of the GSDop:

$$
\mathrm{GSD}_{\mathrm{GP}}=\frac{\mathrm{GSD}_{\mathrm{OP}}}{\sin (\varepsilon)}
$$

where $\quad \varepsilon=$ LOS elevation angle measured from ground plane

The definition of GSD in the Ground Plane (GP) is applicable to satellite sensors which detect targets at high elevation angles; GIQE 4 was derived using images captured at elevation angles between $25^{\circ}$ and $90^{\circ}$ (Harrington, 2015).

Equation 3 represents the sampling distance in the horizontal ground plane, so it does not describe the vertical sampling distance, which becomes increasingly meaningful for interpretability purposes as the elevation angle decreases.

The implication of these definitions is that NIIRS computed by GIQE 3 will not depend on the elevation angle whereas NIIRS computed by GIQE 4 will decrease as this angle decreases.

The GSD may be different in the Along Scan (AS) and Cross Scan (CS) directions; GSD employed in GIQE 3 and GIQE 4 is 
the geometric mean of the GSDs computed in these two directions. In what follows we assume that these two directions are orthogonal, so:

$$
\mathrm{GSD}_{3}=\sqrt{\mathrm{GSD}_{\mathrm{OP}-\mathrm{AS}} \cdot \mathrm{GSD}_{\mathrm{OP}-\mathrm{CS}}}
$$

When one of these two directions makes an elevation angle $\varepsilon$ with the ground plane, the other direction is orthogonal to this plane, thus for GIQE 4:

$$
\mathrm{GSD}_{4}=\frac{\mathrm{GSD}_{3}}{\sqrt{\sin (\varepsilon)}}
$$

\subsubsection{Relative Edge Response}

The Relative Edge Response (RER) is the slope of the normalized system Edge Response (ER) measured between two points located half a pixel from the edge (Fiete, 2010):

$$
\operatorname{RER}=\operatorname{ER}(0.5)-\operatorname{ER}(-0.5)
$$

The ER is normalized in the range from 0 to 1 , as depicted in Figure 1, where the edge of the scene has a dark left side with value 0 and a bright right side with value 1 . Figure 1 illustrates the ER of a raw image, without Modulation Transfer Function Compensation (MTFC) to sharpen the image. The raw image is characterized by a sharpening filter gain $\mathrm{G}=1$.

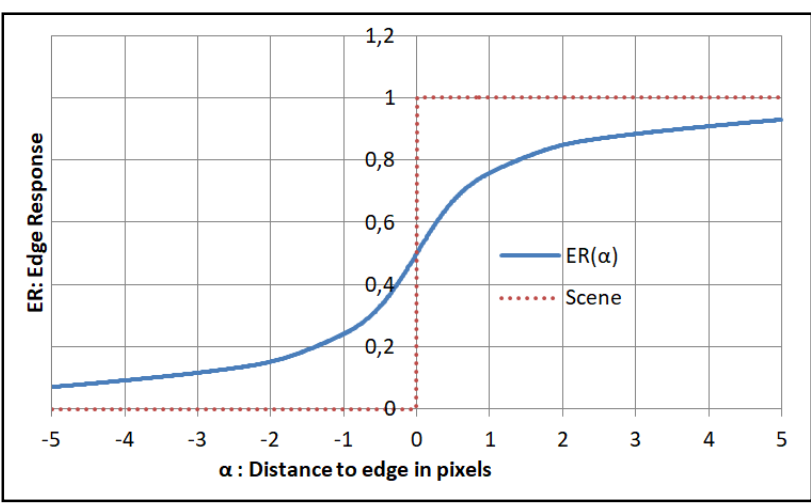

Figure 1. Edge response without MTFC sharpening $(\mathrm{G}=1)$

The ER is measured in the AS and CS directions using artificial or natural targets (Lee, 2014), it can also be computed in terms of the system Modulation Transfer Function (MTF) as (Fiete, 2010) (Auelmann, 2012):

$$
\operatorname{ER}(\alpha)=0.5+\int_{0}^{\frac{1}{Q}}\left[\frac{\sin (2 \pi \chi \alpha)}{\pi \chi}\right] \operatorname{MTF}(\chi) d \chi
$$

where $\quad \alpha=$ distance to the edge in pixel units

$\mathrm{Q}=$ optical factor defined below

$\chi=$ spatial frequency

Optical factor $\mathrm{Q}$ is the ratio between spatial cutoff frequencies of the aperture and the detector, defined as (Fiete, 2010):

$$
\mathrm{Q}=\frac{\lambda}{\mathrm{d}} \cdot \frac{\mathrm{f}}{\mathrm{p}}
$$

where $\quad \lambda=$ mean detector wavelength

$\mathrm{d}=$ optical aperture diameter

In the presentation of GIQE 4 it is mentioned that it " may not be valid" when $\mathrm{Q}>1$ (Leachtenauer, 1997). Despite this warning,
GIQE 4 is employed for $\mathrm{Q}>1$. Successful application of this equation to systems with $\mathrm{Q}$ between 0.43 and 1.77 suggests that GIQE 4 "can be more widely applicable to a broader class of imaging systems than anticipated" (Wong, 2014).

The RER is directly computed as:

$$
\operatorname{RER}=2 \cdot \int_{0}^{\frac{1}{Q}}\left[\frac{\sin (\pi \chi)}{\pi \chi}\right] \operatorname{MTF}(\chi) d \chi
$$

The MTF, the ER and the RER are usually different in the AS and CS directions, the RER value employed in GIQE 3 and 4 is:

$$
\mathrm{RER}_{3}=\mathrm{RER}_{4}=\sqrt{\mathrm{RER}_{\mathrm{AS}} \cdot \mathrm{RER}_{\mathrm{CS}}}
$$

The RER of GIQE 3 and 4 can be either enhanced (with MTFC) or unenhanced. For example: in Figure 1 the unenhanced RER $=0.339$, whereas in Figure 2 the enhanced RER $=0.923$.

\subsubsection{Height Overshoot}

The height overshoot $\mathrm{H}$ is included in GIQE 3 and 4 to penalize the overshoot caused by MTFC. The parameter $\mathrm{H}$ is derived from the ER using the following rules which are somehow at odds with common practice:

1. $\mathrm{H}$ is measured between 1 to 3 pixels from the edge, at 0.25 discrete pixel increments ( 9 measurement points).

2. $\quad \mathrm{H}$ is defined as the full height of the ER whereas in common practice the overshoot, as its name implies, is the excess response with respect to the ideal response.

3. If the ER increases monotonically then $\mathrm{H}$ is defined as the value of the ER at 1.25 pixels from the edge. In the example of Figure 1, $\mathrm{H}=\mathrm{ER}(1.25)=0.786$.

4. If the ER oscillates in amplitude, as depicted in Figure 2, then $\mathrm{H}$ is defined by the maximum ER value at the nine measurement points. In Figure 2, $\mathrm{H}=\mathrm{ER}(1)=1.003$. In this study, the ER was considered to oscillate when its minimum slope is negative $(\mathrm{dER} / \mathrm{d} \alpha<0)$.

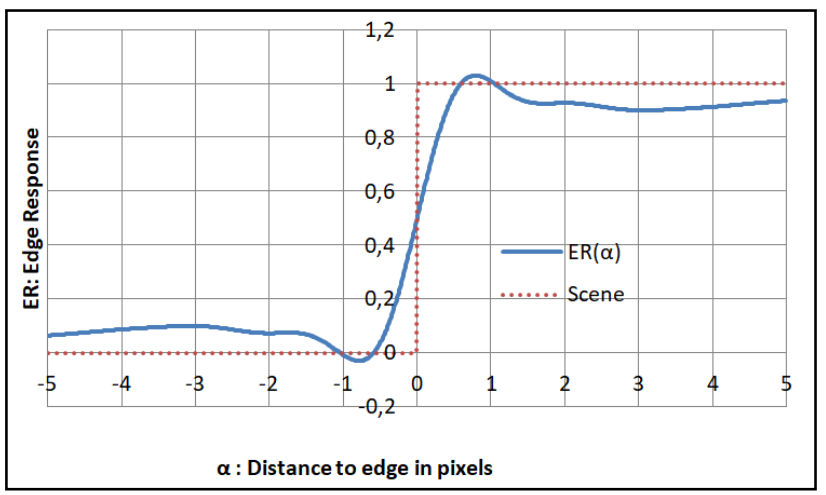

Figure 2. Edge response with MTFC sharpening $(\mathrm{G}=6)$

Rule 1 implies that the maximum overshoot may not be reflected in $\mathrm{H}$, either because; it occurs before one pixel from the edge, as depicted in Figure 2; it occurs after three pixels from the edge, or; it does coincide with the discrete measurement points.

Rules 3 and 4 generate a discontinuity on $\mathrm{H}$ when the ER changes its behaviour from monotonic to oscillatory; this effect is illustrated in Figures 3, 4 and 6 at $\mathrm{G}=2.8$. As illustrated in Figures 4 and 6 , the impact of this discontinuity on the NIIRS rating is minor, more pronounced in GIQE 3 than GIQE 4 due to 
the difference in their D coefficients. This small discontinuity will be tolerated and no attempt will be made to correct it.

Confusion may arise when using GIQE 3 or 4 to compute the NIIRS rating of the raw image, as some users delete the $\mathrm{H}$ term (Wong, 2014). This deletion substantially increases the NIIRS rating; in the example of Figure 1, NIIRS rating of GIQE 3 or 4 is increased by 1.2 or 0.5 points, respectively.

The argument to support this deletion is that the $\mathrm{H}$ term penalizes sharpening and so it is not applicable if no sharpening is used. Although this argument may sound plausible, the assumption that $\mathrm{H}=0$ for raw images is not acceptable because the NIIRS rating will then be a discontinuous function at $\mathrm{G}=1$, as an image with an infinitesimal amount of sharpening $(\mathrm{G} \approx 1)$ will have much less interpretability than the raw image $(G=1)$.

The height overshoot is separately computed in the AS and CS directions, the value that must be employed in GIQE 3 and 4 is:

$$
\mathrm{H}=\sqrt{\mathrm{H}_{\mathrm{AS}} \cdot \mathrm{H}_{\mathrm{CS}}}
$$

\subsubsection{Sharpening Filter Gain}

This parameter penalizes the increase in noise due to MTFC; to evaluate raw images $\mathrm{G}=1$ must be employed.

For the $3 \times 3$ and $5 \times 5$ sharpening filters assumed in the GIQE, the filter gain is computed as the root of the sum of the squares of the MTFC kernel values. The kernel of the $3 \times 3$ convolution filter used in this study is (Auelmann, 2012):

$$
\text { kernel }=\left[\begin{array}{lll}
c & b & c \\
b & a & b \\
c & b & c
\end{array}\right]
$$

The so called "noise gain" or "processing gain" is:

$$
G=\sqrt{a^{2}+4 \cdot b^{2}+4 \cdot c^{2}}
$$

The normalizing condition requires that the sum of all the kernel values is equal to $1(\mathrm{a}+4 \cdot \mathrm{b}+4 \cdot \mathrm{c}=1)$.

Figure 2 was obtained by applying a $3 \times 3$ kernel with $G=6$ to the ER of Figure 1. To compute the kernel values as a function of $G$, the following equations were used:

$$
\mathrm{b}=\mathrm{k} \cdot \mathrm{c}
$$

where $\quad \mathrm{k}=$ given proportionality coefficient

$$
\begin{aligned}
& m=\frac{1+k^{2}}{4 \cdot(1+k)^{2}} \\
& \mathrm{a}=\frac{\mathrm{m}+\sqrt{\mathrm{G}^{2} \cdot(1+\mathrm{m})-\mathrm{m}}}{1+\mathrm{m}} \\
& \mathrm{c}=\frac{1-\mathrm{a}}{4 \cdot(1+\mathrm{k})}
\end{aligned}
$$

To compute the kernel values for a given value of $\mathrm{k}$, the previous equations must be successively applied in the following order; $15,16,17$ and 14 .

A value $k=3$ was used in this study.

\subsubsection{Signal Difference to Noise Ratio}

The Signal Difference to Noise Ratio (SDNR) is computed as the ratio of the signal difference between two lambertian surfaces and the noise. The two surfaces have reflectances $\rho=0.15$ and $\rho=0.07$. The noise is usually computed for the worst case, which is; for the highest reflectance (Fiete, 2001). With these conventions:

$$
\operatorname{SDNR}=\frac{S(\rho=0.15)-S(\rho=0.07)}{N(\rho=0.15)}
$$

where $\quad S(\rho)=$ signal for Lambert target with reflectance $\rho$ $\mathrm{N}(\rho)=$ noise for Lambert target with reflectance $\rho$

The Signal to Noise ratio (SNR) for a target with reflectance $\rho$ is defined as:

$$
\operatorname{SNR}(\rho)=\frac{S(\rho)}{N(\rho)}
$$

The computation of the SDNR requires detailed knowledge of the sensor, the atmospheric transmission path and all the noise contributions.

The following rough relationship between $\operatorname{SNR}(\rho)$ and the GIQE SDNR, is obtained assuming that SNR is proportional to the square root of the signal $\mathrm{S}$ (photon noise predominates) and that this signal is directly proportional to target reflectance:

$$
\operatorname{SDNR} \approx \operatorname{SNR}(\rho) \cdot \frac{0.08}{\sqrt{0.15 \cdot \rho}}
$$

The SDNR is approximately equal to the SNR if $\rho=0.04$, a case rarely encountered in practice. If $\rho=1$ equation 20 predicts that the SNR is five times larger than the SDNR, a more accurate model indicates that, under this same condition, it can be six to ten times larger (Fiete, 2001).

The SDNR of the GIQE is denominated "SNR", a practice that leads to confusion between this GIQE parameter and the Signal to Noise Ratio of the image. We have used SDNR instead, to minimize further confusion on this issue.

\subsection{GIQE 5}

GIQE 5 predicts the NIIRS rating of processed images, without specifying the processing technique, as a function of the raw image parameters. This equation has the form:

$$
\begin{aligned}
\text { NIIRS }_{5}= & A+\mathrm{B} \cdot \log \left(\mathrm{GSD}_{5}\right)+ \\
& \mathrm{C} \cdot[1-\exp (\mathrm{D} / \mathrm{SDNR})] \cdot \log \left(\mathrm{RER}_{5}\right)+ \\
& \mathrm{E} \cdot\left[\log \left(\mathrm{RER}_{5}\right)\right]^{4}+\frac{\mathrm{F}}{\mathrm{SDNR}}
\end{aligned}
$$

where $\mathrm{A}, \mathrm{B}, \mathrm{C}, \mathrm{D}, \mathrm{E}, \mathrm{F}=$ constants defined below $\mathrm{GSD}_{5}=$ Ground Sampling Distance used in GIQE 5 $\mathrm{RER}_{5}=$ Unenhanced (without MTFC) Relative Edge Response used in GIQE 5

The constants are defined in Table 3 assuming that the GSD is expressed in meters.

\begin{tabular}{|c|c|c|c|c|c|c|}
\hline GIQE & A & B & C & D & E & F \\
\hline 5 & 4.274 & -3.32 & 3.32 & -1.9 & -2.0 & -1.8 \\
\hline
\end{tabular}

Table 3. GIQE 5 coefficients for GSD in metric units

We assumed that the "SNR" of GIQE 5, is the same SDNR of GIQE 3 and 4. This assumption is coherent with the relatively 
low SDNR values of Table 4 and with the explicit definition of "SNR" in the preliminary version of GIQE 5 (Griffith, 2012)

GIQE 5 coefficients were derived by regression analysis of a set of 49 airborne images which covered the range of parameters indicated in Table 4 (Harrington, 2015).

\begin{tabular}{|c|c|c|c|}
\hline Parameter & Minimum & Maximum & Mean \\
\hline $\mathrm{GSD}_{\mathrm{GP}}(\mathrm{m})$ & 0.08 & 1.4 & 0.47 \\
\hline RER & 0.31 & 0.54 & 0.41 \\
\hline SDNR & 4.1 & 26.7 & 10.6 \\
\hline NIIRS & 2.9 & 7.7 & 5.9 \\
\hline $\mathrm{Q}$ & 1 & 2 & Unknown \\
\hline$\varepsilon$ & 8.9 & 87.3 & 34.6 \\
\hline
\end{tabular}

Table 4. GIQE 5 range of parameters

The GSD and RER parameters of GIQE 5 are defined in a special way as indicated in what follows.

\subsubsection{Ground Sampling Distance}

GIQE 5 considers the benefit of both vertical and horizontal resolution by defining GSD as the geometrical mean of $\mathrm{GSD}_{3}$ and $\mathrm{GSD}_{4}$. With the same assumptions and nomenclature of section 2.1.1 the GSD of GIQE 5 is:

$$
\mathrm{GSD}_{5}=\frac{\mathrm{GSD}_{3}}{\sqrt[4]{\sin (\varepsilon)}}
$$

\subsubsection{Relative Edge Response}

To incorporate smear, GIQE 5 assumes that $\mathrm{RER}_{\mathrm{CS}} \geq \mathrm{RER}_{\mathrm{AS}}$ and defines the RER to be used as:

$$
\mathrm{RER}_{5}=\sqrt[3]{\mathrm{RER}_{\mathrm{CS}} \cdot \mathrm{RER}_{\mathrm{AS}}^{2}}
$$

This equation "implies that a loss of RER in one direction cannot be mitigated by a simple proportional increase of RER in the orthogonal direction." (Harrington, 2015).

The movement along the scan plane degrades only the $\mathrm{MTF}_{\mathrm{AS}}$ so the usual case is that RER $\mathrm{RS}_{\mathrm{C}} \geq \mathrm{RER}_{\mathrm{AS}}$, but, if some factors that deteriorate mainly the $\mathrm{MTF}_{\mathrm{CS}}$ prevail, such as charge transfer efficiency, then RER $\mathrm{RS}_{\mathrm{CS}}<\mathrm{RER}_{\mathrm{AS}}$. Examples of images in with $\mathrm{MTF}_{\mathrm{CS}}>\mathrm{MTF}_{\mathrm{AS}}$ have been presented for Cartosat -1 sensors (Crespi, 2009).

To accommodate the possibility that $\mathrm{RER}_{\mathrm{CS}}<\mathrm{RER}_{\mathrm{AS}}$, equation 23 must be generalized to:

$$
\operatorname{RER}_{5}=\sqrt[3]{\mathrm{RER}_{\mathrm{X}} \cdot \mathrm{RER}_{\mathrm{Y}}^{2}}
$$

Where RER $\mathrm{X} \geq \mathrm{RER}_{\mathrm{Y}}$ and the $\mathrm{X}$ and $\mathrm{Y}$ directions can be either AS or CS, depending on their relative RER values.

Equation 24 reduces to equation 23 when $R R_{C S} \geq R R_{A S}$, but when $R_{\text {ER }}<\mathrm{RER}_{\mathrm{AS}}$ it gives more weight to the lesser value, as required by the previous quote.

\subsection{Continuous Form of GIQE 4}

\subsubsection{Generalities}

We refer here only to the discontinuity due to the two forms of GIQE 4 (GIQE 4a and GIQE 4b) and neglect the minor discontinuity, due to $\mathrm{H}$, mentioned above at 2.1.3. The former discontinuity of GIQE 4 at RER $=0.9$ has been already noted (Auelmann, 2012) (Griffith, 2012).

At the point of discontinuity (RER=0.9) GIQE 4a (RER < 0,9) predicts greater NIIRS rating than GIQE $4 b$ (RER $\geq 0.9)$. The discontinuity is negligible ( 0.02 NIIRS points) for $\mathrm{GSD}=8 \mathrm{~cm}$ but it increases to 0.24 points for GSD $=2 \mathrm{~m}$.

GIQE 3 predicts that when the noise gain $G$ is increased the NIIRS rating also increases until a maximum rating is achieved; further increase of $\mathrm{G}$ will deteriorate the rating due to excessive values of noise amplification and overshoot. This behaviour is illustrated in Figure 4 by the upper blue curve.

GIQE 4 predicts a first maximum just below $\mathrm{RER}=0.9$ and a local minimum at RER $=0.9$. For RER $>0.9$ it displays the same behaviour of GIQE 3, achieving a second maximum at roughly the same gain for which the maximum of GIQE 3 is achieved. This behaviour is illustrated in Figure 4 by the lower red curve.

The first spurious maximum of GIQE 4 is popular among vendors as it offers the theoretical possibility of achieving, with moderate noise gain, a maximum NIIRS rating that can only be - eventually - surpassed with a much higher noise gain.

\subsubsection{Reference Sensor}

For this comparative study we will use the same reference sensor considered in the study of GIQE 5 , characterized by a circular aperture whose central obstruction blocks $20 \%$ of the aperture (linear obstruction ratio 0.447), a Wave Front Error (WFE) of 0.1 wavelengths and an array of square detectors with $100 \%$ fill factor (Harrington, 2015). The MTF of this sensor is well known (Cota, 2010) so its ER can be directly obtained using equation 7. The ER of this sensor with optical factor $Q=1$, is illustrated in Figure 1 for $\mathrm{G}=1$ and in Figure 2 for $\mathrm{G}=6$.

We neglect movement and smear so, conveniently for our comparison purposes, if $\mathrm{G}=1$, then $\mathrm{RER}_{3}=\mathrm{RER}_{4}=\mathrm{RER}_{5}=$ RER $_{\mathrm{AS}}=\mathrm{RER}_{\mathrm{CS}}=\mathrm{RER}$. We also assume that $\mathrm{GSD}_{3}=\mathrm{GSD}_{4}=$ $\mathrm{GSD}_{5}=\mathrm{GSD}$.

Our reference sensor differs slightly from the one defined in the GIQE 5 paper (Harrington, 2015); we assume a monochromatic WFE instead of a polychromatic WFE, so our RER for $\mathrm{G}=1$ is $\operatorname{RER}_{0}=0.339 \approx 0.34$ instead of $\mathrm{RER}_{0}=0.329$.

\subsubsection{Nature of the discontinuity}

In Figure 3 the RER and $\mathrm{H}$ of the reference system are presented as a function of the noise gain for $Q=1$. As expected $H$ is discontinuous at the minimum $\mathrm{G}$ value that presents a negative ER slope $(G=2.8$ for the case of Figure 3$)$.

In Figure 4 the NIIRS values predicted by GIQE 3, 4 and 5 for this reference system are presented versus $\mathrm{G}$ for $\mathrm{Q}=1, \mathrm{GSD}=2$ $\mathrm{m}$ and $\mathrm{SDNR}=30$. GIQE 5 prediction is presented as a slashed horizontal line to emphasize that it represents a limiting value that does not depend on the noise gain.

GIQE 3 predicts that the optimum gain which maximizes NIIRS is $\mathrm{G}_{\text {opt }} \approx 12$. GIQE 4 a predicts instead $\mathrm{G}_{\text {opt }}=5.8$, but this sharp optimum is considered a spurious result generated by the discontinuity at $\mathrm{ER}=0.9$. GIQE $4 \mathrm{~b}$ predicts $\mathrm{G}_{\mathrm{opt}} \approx 13$, which correlates much better with the $\mathrm{G}_{\text {opt }}$ of GIQE 3 . 


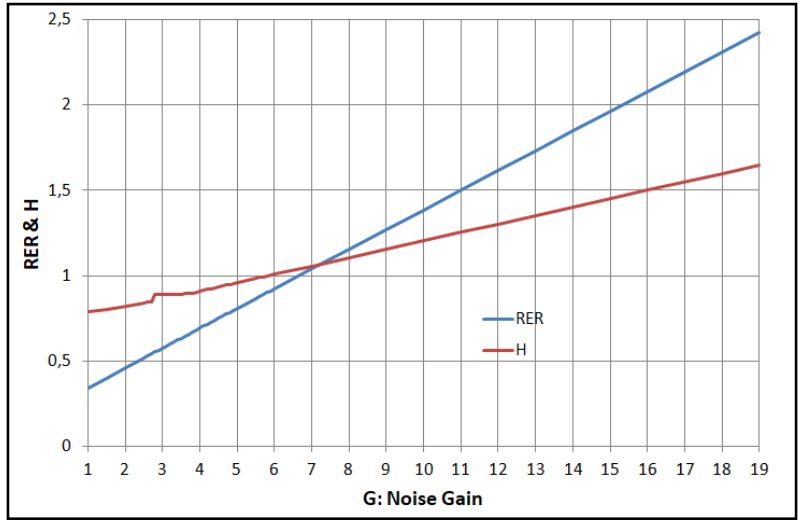

Figure 3: RER and $\mathrm{H}$ versus $\mathrm{G}$ for $\mathrm{Q}=1$ reference sensor

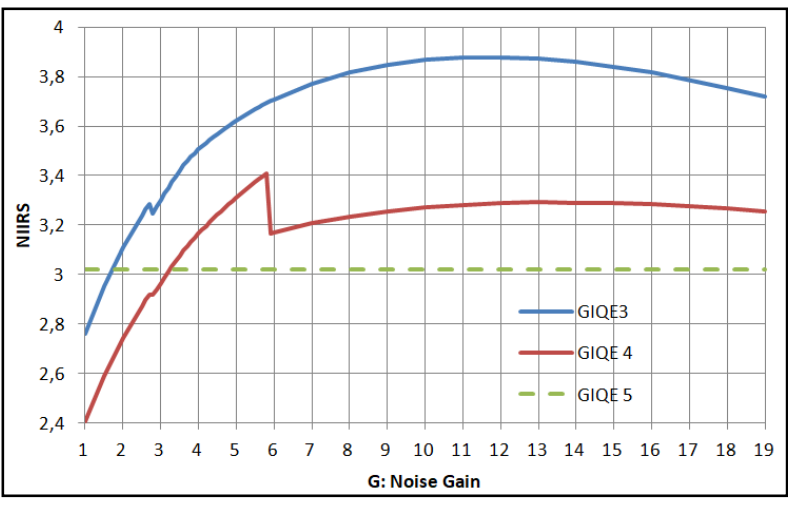

Figure 4: NIIRS for ideal $\mathrm{Q}=1$ system with $\mathrm{SDNR}=30$

The spurious character of the optimum predicted by GIQE $4 \mathrm{a}$ is more evident when the NIIRS rating of GIQE 5 is considered. The discrepancy between GIQE 4 and GIQE 5 can be reduced by deriving a continuous form of GIQE 4 that displays a single optimum gain.

\subsubsection{Continuous form of GIQE 4}

The goal is to derive a GIQE $4 \mathrm{C}$ equation which is as similar as possible to GIQE 4 but that is continuous at RER $=0.9$. The sources of GIQE 4 discontinuity are the coefficients A, B and C of equation 1 that change abruptly at RER $=0.9$ (see Table 1 ).

We consider first the $\mathrm{A}$ and $\mathrm{C}$ coefficients and concentrate on the functions $f(R E R)=A+C \cdot \log (R E R)$. Figure 5 presents the $f_{a}$ and $f_{b}$ functions for GIQE $4 \mathrm{a}$ and $4 \mathrm{~b}$, respectively.

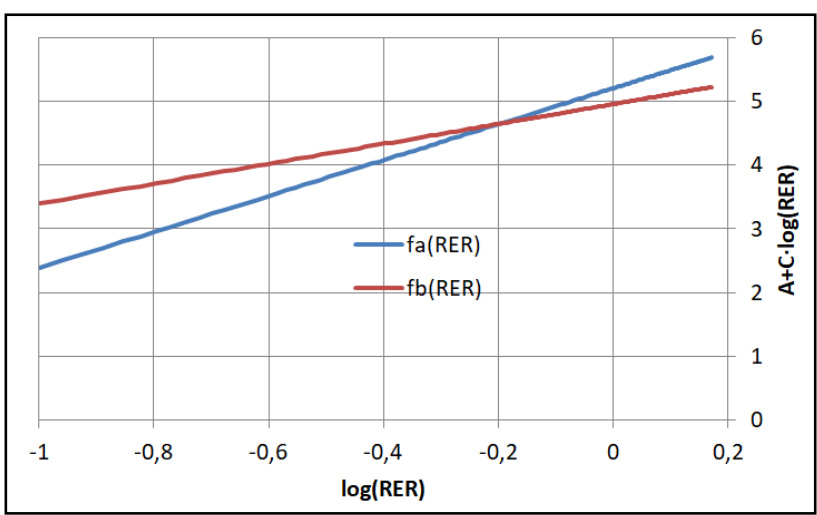

Figure 5: Functions $\mathrm{f}_{\mathrm{a}}(\mathrm{RER})$ and $\mathrm{f}_{\mathrm{b}}(\mathrm{RER})$ for GIQE 4
The most simple way to eliminate the discontinuity due to A and $\mathrm{C}$ coefficients is to choose the first transition point at the intersection of $f_{a}$ and $f_{b}$ which occurs at $\log (0.63)=-0.20$.

To eliminate the discontinuity of the B coefficient we define a transition region in the range $0.63<\mathrm{RER}<0.9$, and postulate in this region a variable $\mathrm{B}$ coefficient of the form:

$$
\mathrm{B}(\mathrm{RER})=-3.32+\frac{(0.9-\mathrm{RER})}{0.27} \cdot 0.16
$$

This equation was derived by requiring that the B coefficient is continuous at the two edges of the transition region. The final form of the continuous GIQE 4C equation is then:

1. For RER $\leq 0.63$ as in GIQE 4a.

2. For $0.63 \leq \mathrm{RER} \leq 0.9$ as in GIQE $4 \mathrm{~b}$ but replacing $\mathrm{B}=-3.32$ with $\mathrm{B}(\mathrm{RER})$ as given by equation 25 .

3. For RER $\geq 0.9$ as in GIQE $4 b$.

With respect to GIQE 4 we have modified only GIQE 4 a for RER > 0.63, leaving GIQE $4 \mathrm{~b}$ unscathed. Apparently it would have been more elegant to define a transition region centred at RER $=0.9$ and define variable $\mathrm{A}, \mathrm{B}$ and $\mathrm{C}$ coefficients as in equation 25 , but this alternative procedure does not remove, it only reduces, the artificial maximum near RER $=0.9$.

GIQE 4C is presented in Figure 6 for the same conditions indicated in Figure 4. As expected from Figure 5, there is a discontinuity of the slope at the point of transition $\mathrm{RER}=0.63$ $(\mathrm{G}=3.5$ in Figure 6).

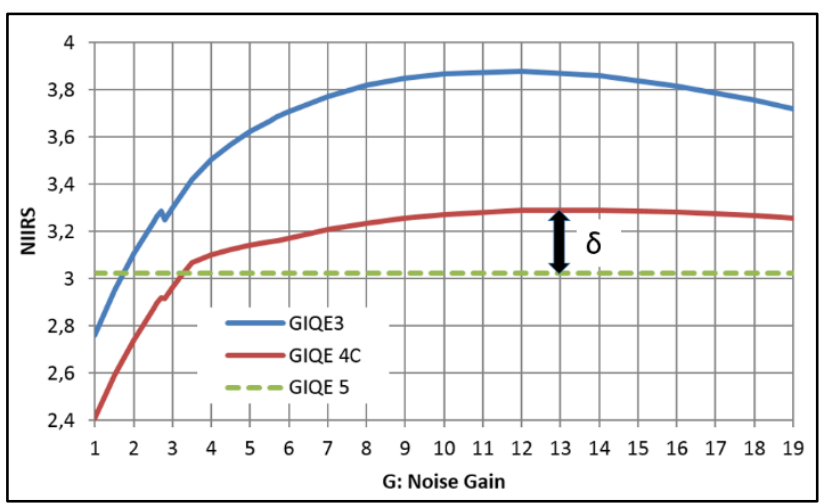

Figure 6: As Figure 4 with continuous form of GIQE 4

To validate the derivation of GIQE $4 \mathrm{C}$ we note the following six facts, the first three are of general nature whereas the last three are particular properties of the reference sensor:

1. The abrupt discontinuity of the function at RER $=0.9$ has been completely eliminated.

2. Figure 4 suggests that GIQE $4 \mathrm{~b}$ is behaving properly near RER $=0.9$ whereas GIQE $4 \mathrm{a}$ is going astray. This is the first reason to leave GIQE $4 \mathrm{~b}$ unscathed.

3. GIQE $4 \mathrm{~b}$ follows the "common NIIRS policy" that requires $\mathrm{B}=-3.32$, whereas GIQE 4a does not (Harrington, 2015). This is the second reason to leave GIQE $4 \mathrm{~b}$ unscathed.

4. The discrepancy between the maximum NIIRS ratings predicted by GIQE 4 and GIQE 5 is 0.48 . GIQE 4 C has reduced this discrepancy to 0.27 .

5. Comparing GIQE 3 with GIQE 4, the optimum gains differ by a factor of 2.1. GIQE $4 \mathrm{C}$ has reduced this discrepancy to a factor of 1.1 
6. The root mean square difference between GIQE 4 and GIQE 4C within the transition zone is 0.14 NIIRS, which is less than half the standard error of GIQE 4.

With these facts we validate the use of GIQE 4 C to replace GIQE 4 , removing the spurious NIIRS maximum associated to the discontinuity at RER $=0.9$. We consider now the former version to compute the maximum NIIRS rating that can be achieved by image processing.

\section{COMPARATIVE STUDY}

\subsection{Generalities}

We accept GIQE 5 as the ultimate version and compute the overestimation error " $\delta$ " incurred by GIQE 4C users, when computing the maximum NIIRS rating of a processed image, This error is illustrated in Figure 6 and defined as:

$$
\delta=\mathrm{NIIRS}_{4 \mathrm{C}}(\mathrm{G}=\mathrm{Gopt})-\mathrm{NIIRS}_{5}
$$

In what follows we consider GSD $=1$ meter, the error $\delta$ is independent of the GSD when the optimum gain occurs for a processed RER $\geq 0.9$, because in this case the coefficients $B$ of GIQE 4C and GIQE 5 are equal. This independence condition is fulfilled for the two lower red and blue curves of Figure 7.

In this comparative study we computed $\delta$ as a function of SDNR for three different values of the unenhanced RER; the minimum value considered by GIQE $4\left(\mathrm{RER}_{0}=0.2\right)$, the maximum value considered by GIQE $5\left(\operatorname{RER}_{0}=0.54\right)$ and the value for the reference $\mathrm{Q}=1$ sensor previously considered $\left(\mathrm{RER}_{0}=0.34\right)$.

The technique to change the RER of a sensor by varying its value of $\mathrm{Q}$ has already been used in the context of GIQE studies (Thurman, 2008). We use this same technique and consider the same reference sensor with $\mathrm{Q}=0.35$ to achieve an unenhanced $\mathrm{RER}_{0}=0.539 \approx 0.54$ and with $\mathrm{Q}=2.17$ to achieve an unenhanced $\operatorname{RER}_{0}=0.2001 \approx 0.2$.

\subsection{Results}

Figure 7 presents the overestimation error $\delta$ of GIQE 4C when computing the maximum NIIRS rating of a processed image, for the three previous values of unenhanced RER, assuming the reference sensor specified at 2.3.2, with GSD $=1$ meter. The $\delta$ value illustrated in Figure 6 is here represented as a small circle.

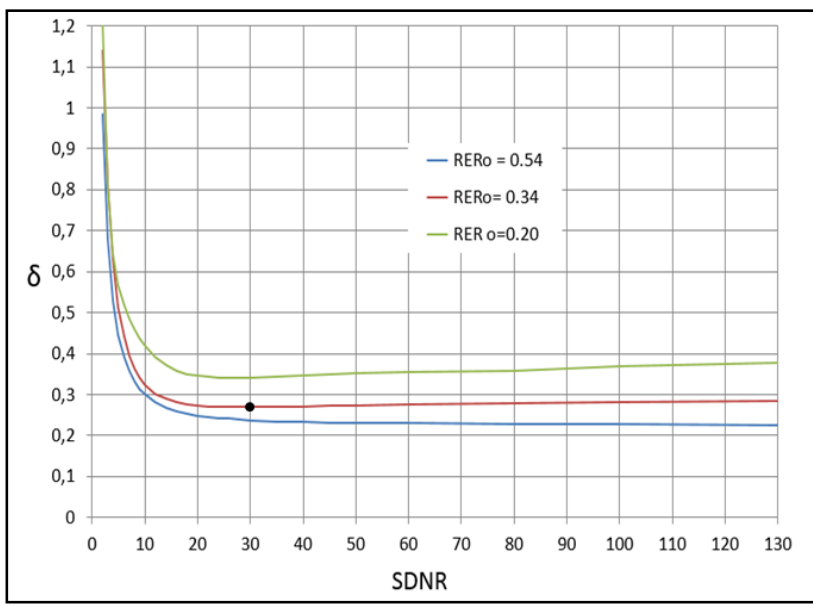

Figure 7: NIIRS error of GIQE 4C for reference system
This error has been plotted for the whole range of SDNR values considered in the derivation of GIQE 4 (Table 2). The error $\delta$ is excessive if SDNR $<8$. This result is coherent with the observations that GIQE 4 is inaccurate for low SDNR values (Thurman, 2008) and that that for low SNR the quality of the raw image is better than the one of the processed image (Auelmann, 2012).

For larger SDNR the error will depend on the value of $\mathrm{RER}_{0}$; for SDNR > 20 a somewhat asymptotic behaviour is achieved.

The smallest error is obtained for the largest $\mathrm{RER}_{0}$, presumably because in this case the raw image has better quality and the benefit of image processing is smaller. Even in this favourable case the error $\delta$ is important being larger than twice the interpretability threshold (0.1 NIIRS).

For the intermediate $\mathrm{RER}_{0}$ value the error $\delta$ is slightly less than the GIQE standard error, casting doubts on the applicability of GIQE 4C under this condition.

For the smaller RER $\mathrm{R}_{0}$ the error $\delta$ is larger than GIQE standard error, severely limiting the validity of GIQE $4 \mathrm{C}$ for this case.

If GIQE 4 is used instead of GIQE 4C the three previous overestimation errors increase roughly 0.2 NIIRS, justifying the use of the continuous form of this equation.

\section{DISCUSSION}

GIQE 3 and GIQE 4 were developed to evaluate NIIRS rating of raw or processed hardcopy images, as a function of five parameters; GSD, RER, H, SDNR and G. Two of these parameters (RER and $\mathrm{G}$ ) depend on the image processing technique, so these equations can be used to compute the optimum processing gain $\mathrm{G}$ that maximizes image quality.

One drawback of GIQE 4 is the discontinuity at RER $=0.9$ that generates a spurious NIIRS maximum at moderate processing gains. This maximum has been removed deriving a continuous form of this equation (GIQE 4C) that displays good correlation with GIQE 3 regarding the optimum processing gain.

Before the advent of GIQE 5 some authors using GIQE 4 stated that "removing the post-processing process in the GIQE will allow a conservative estimation of the system's technical specifications, ensuring the system will deliver adequate performance", paving the way to GIQE 5. Coherently, with this posture, some satellite manufactures of high quality imagers specified only their raw image GIQE 4 NIIRS rating, disregarding the processing improvement. On the contrary, other manufacturers claimed improvements of up to 1.0 NIIRS point due to image sharpening.

GIQE 5 represents a "paradigm shift" on NIIRS prediction using raw image parameters to rate the quality of a "well processed" image. Two parameters of GIQE 3 and 4 (H and G) are removed and a more complex mathematical form that gives more weight to the SDNR is postulated (Harrington, 2015).

A formal protest was issued in 2001 by a sensor manufacturer against a military procurement office, based among other issues, on the claim that GIQE 4 provided insufficient weight to the signal to noise ratio (Barton, 2001). Although this protest was denied, GIQE 5 gives some late credit to it. 
Although GIQE 5 considers raw image parameters, it gives no clue on the quality of the raw image, so it cannot be used to compute the improvement on image quality due to image processing or to estimate the optimum processing gain. For these purposes, GIQE 4 may still be used, even though it has been declared "obsolete" with GIQE 5 appearance (Harrington, 2015). To salvage some applicability of GIQE 4 under this obsolescence dictum its continuous form is preferred, using GIQE 5 predictions to define the maximum NIIRS rating of processed images.

This paper compared the optimum NIIRS predictions of GIQE 4C with those of GIQE 5. To perform this comparison some reference sensor needs to be defined, for the sake of brevity, only the sensor defined in GIQE 5 presentation was here considered. The study of different sensors is a matter of future research.

Current results show the following general trends:

1. GIQE 4 overestimates the benefit of image processing on image quality.

2. GIQE 4 underestimates the degradation on image quality due to low signal difference to noise ratio.

3. The two previous GIQE 4 errors get progressively larger as the raw RER decreases.

It has been noted that the NIIRS rating of submetric satellite raw images strongly correlates with their in orbit spatial resolution measurements (Valenzuela, 2019). The relationship between GIQE 5 and spatial resolution is a matter of future research.

\section{CONCLUSIONS}

The three known versions of the General Image Quality Equation (GIQE) have been presented and analysed against a common reference framework.

The subtleties, peculiarities, limitations and common pitfalls in the use of these equations have been highlighted and some solutions to the more common problems found in their application have been suggested. In particular a continuous form of GIQE 4, denominated GIQE 4C, has been derived to remove its main logical inconsistency and this new form has been validated against the predictions of GIQE 3 and GIQE 5.

A comparative study between GIQE 4C and GIQE 5 has been performed, based on the reference sensor used in the presentation of this last equation. The results of this study indicate that GIQE $4 \mathrm{C}$, and GIQE 4 to a greater extent, overestimate the maximum image quality that can be obtained by image processing.

The NIIRS overestimation error of GIQE 4 is particularly large with low SDNR, for the whole range of RER $_{0}$ values considered in GIQE 5 derivation. These errors are also excessive for small $\mathrm{RER}_{0}$ values even under high SDNR conditions. Despite these errors, GIQE 4 may still be useful with some restrictions.

GIQE 5 has generated a paradigm shift on image quality metrics leading GIQE 4 to obsolescence. This new metric reduces the benefit of image processing and enhances the importance of the raw image and its signal to noise ratio.

\section{ACKNOWLEDGEMENTS}

The authors express their gratitude to the following institutions and individuals that made this work possible:
1. To the Chilean Air Force for financing, supporting and stimulating this work.

2. To the aerospace companies that provided the Chilean Ministry of Defense with information about the NIIRS rating computation of their satellite sensors, during the Request For Information (RFI) of the "Catalejo" project.

3. To the reviewers for providing helpful suggestions for improving the clarity, structure and content of this paper.

\section{REFERENCES}

Auelmann, R., 2012. Image Quality Metrics. http://www.techarchive.org/wp-content/themes/boilerplate/ largerdocs/Image\%20Quality\%20Metrics.pdf

Barton et al, 2001, Decision, United States General Accounting Office, Matter of: Recon-Optical, Inc., File: B-286529, Date: January 18, 2001.

Cota, S. A. et al., 2010. PICASSO: an end-to-end image simulation tool for space and airborne imaging systems. Journal of Applied Remote Sensing, Vol. 4, 043535, pp. 1-36.

Crespi, M. and De Vendictis L., 2009. A procedure for high resolution satellite imagery quality assessment. Sensors, vol. 9, No. 5, pp. 3289-3313.

Fiete, D. R and Tantalo, T., 2001. Comparison of SNR image quality metrics for remote sensing systems. Opt. Eng. Vol. 40 No. 4, pp. 574-585.

Fiete, R. D., 2010. Modeling the imaging chain of digital cameras. Belligham WA: SPIE Press, Oct. 2010.

Griffith, D., 2012. General Image Quality Equation (GIQE). National Geospatial-Intelligence Agency. https://calval.cr.usgs.gov/wordpress/wp-content/uploads/ Griffith_Doug_JACIEGIQERev3cor2sjs6with-Caveat.pdf

Harrington, L. et al, 2015. General Image Quality Equation; GIQE version 5. National Geospatial-Intelligence Agency. http://www.gwg.nga.mil/ntb/baseline/docs/GIQE-5_for_Public_ Release.pdf

Irvine, J. M., 1997. National Imagery Interpretability Rating Scales (NIIRS): Overview and Methodology. SPIE Vol. 3128 No. 0277-786X, pp. 93-103.

Leachtenauer, J. et al, 1997. General Image-Quality Equation: GIQE. Applied Optics, Vol. 36, No. 32, pp. 8322 - 8328.

Lee, D. et al, G., 2014. Spatial Quality for Satellite Image data and Landsat 8 OLI Lunar data. Presented at 38th CEOS Working Group on Calibration and Validation Plenary.

Thurman, S. T. et al, 2008. Analysis of the general image quality equation. Proc. of SPIE, Vol. 6978 69780F, pp. 1-13.

Valenzuela Q. A. and Reyes J. C. G, 2019. Basic spatial resolution metrics for satellite imagers. IEEE Sensors Journal, DOI 10.1109/JSEN.2019.2902512, pp. 1 - 9.

Wong S. et al, 2014. Predicting image quality of surveillance sensors. Defence Research and Development Canada. Scientific Report DRDC-RDDC-2014-R97. 